\title{
Algumas reflexões sobre consumos de substâncias psicoactivas e comportamentos aditivos na população
}

Cristina Ribeiro*

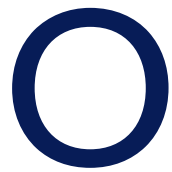
s vários problemas de saúde pública e sociais associados ao consumo de tabaco, do álcool e de substâncias ilícitas exigem uma maior atenção por parte da comunidade, assim como respostas e políticas apropriadas no sentido da sua resolução.

Com base num padrão de medida conhecido como Anos de Vida Ajustados por Incapacidade (DALY), consegue-se avaliar a carga imposta à sociedade por mortes prematuras e anos vividos com incapacidades. $\mathrm{O}$ projecto da Organização Mundial de Saúde «Comparative quantification of health risks: global and regional burden of disease due to selected major risk factors", sobre a carga global das doenças, mostrou que o tabaco e o álcool eram causas importantes de mortalidade e incapacidade em países desenvolvidos. ${ }^{1}$

A expressão «consumo de substâncias» é utilizada como qualquer forma de auto-administração de uma substância psicoactiva. É utilizada em vez de «consumo nocivo de substâncias» como uma expressão mais geral englobando todos os níveis de consumo, incluindo o consumo ocasional ou prolongado destas mesmas substâncias. ${ }^{2}$

Álcool e tabaco são substâncias lícitas largamente disponíveis na maior parte do mundo e cujas campanhas de publicidade proporcionam a sua promoção, 0 que pode ter um efeito negativo quando dirigido a de-

*Professora Auxiliar da Faculdade de Medicina de Lisboa. Médica de Família. Focal point de projectos Europeus na área do álcool e CSP. terminados grupos populacionais, nomeadamente os jovens.

De acordo com o World Health Report 2002 (WHO, 2002), $8,9 \%$ do peso global da doença, medido através do número de anos de vida potencial perdidos ajustados para a incapacidade (disability adjusted life yearsDALY's), advém do uso de substâncias. ${ }^{1,3}$

Nos países desenvolvidos, nomeadamente na região europeia, o consumo de álcool e de tabaco possui níveis e padrões tradicionalmente mais elevados e abrangentes que, apesar de estarem a decrescer nos últimos anos, implicam ainda um maior número de consequências sociais e de saúde do que noutras regiões do globo. ${ }^{3}$

Em relação às drogas ilícitas, a Cannabis é a mais consumida em todos os países da União Europeia, apesar de existirem diferenças de prevalência de consumo. Os dados do estudo ESPAD (European School Survey Project on Alcohol and other Drugs) ${ }^{4}$ são uma importante fonte de informação para o Observatório Europeu da Droga e da Toxicodependência e acompanham a evolução dos consumos de substâncias lícitas e ilícitas nos alunos de 16 anos nos diversos países da Europa. As substâncias lícitas demonstram ter, para além de taxas de prevalência altas, uma continuidade de uso mais persistente em todas as faixas etárias. As taxas de continuidade de uso de algumas drogas ilícitas nas faixas etárias mais jovens são relativamente altas, apesar de corresponderem a prevalências mais baixas. Para além destes dados de consumo, um aspecto preocupante, 
que também nos é revelado pelos inquéritos escolares é a diminuição da percepção de risco por parte da população juvenil com este tipo de substâncias.

\section{ESTRATÉGIAS A IMPLEMENTAR}

Oferecer uma resposta a este problema e promover a mudança de comportamento individual requer a reorientação das acções dos profissionais. Além disso, sendo o consumo de substâncias um comportamento social, o sucesso das intervenções depende em parte de um contexto social existente que lhe seja favorável, o que implica o desenvolvimento de políticas públicas específicas.

Estudos recentes têm demonstrado que todos os profissionais de saúde dos Cuidados de Saúde Primários, em geral, se encontram numa posição única para identificarem ou mesmo intervirem junto de indivíduos que usam substâncias, mesmo que alguns necessitem de ser encaminhados para tratamento especializado. ${ }^{5,6,7,8,9}$

Há que incorporar programas de detecção e intervenção precoce dirigidos a menores com problemas de consumo de álcool e drogas, assim como um olhar atento aos filhos dos dependentes destas substâncias.

Há que valorizar a aplicação de novas abordagens e métodos terapêuticos, em suma, uma actualização com novas soluções face a novos diagnósticos e novos padrões de consumo.

O desenvolvimento das diferentes medidas referidas tem ser implementado a par da investigação e formação de profissionais, bem como a recolha e análise de dados procedentes de diversas fontes que permitam dispor de uma visão o mais precisa possível em cada momento de actuação. É fundamental implementar projectos de investigação epidemiológica, clínica e de saúde pública nos diversos contextos em que existam substâncias psicoactivas implicadas.

\section{MEDIDAS COMUNITÁRIAS}

Influenciar níveis e padrões de consumo de substâncias requer abordagens direccionadas para além do consumidor individual e que abranjam acções comunitárias e de saúde pública. Assim, para além das intervenções direccionadas ao indivíduo em particular e das consequentes acções de governação clínica e de gestão do sistema de saúde e articulação em rede dos serviços de saúde, existe na área do uso de substâncias, a necessidade de se desenvolverem políticas públicas de saúde claras e consistentes. ${ }^{10,11}$ Estas requerem a intervenção de diversos sectores do poder público e da sociedade, nomeadamente na implementação de estratégias relacionadas com:

- Disponibilidade das substâncias na sociedade;

- Acessibilidade das substâncias por parte da população;

- Dissuasão, penalização e regulamentação das condições e dos padrões de uso em contextos sociais específicos;

A limitação da oferta, com especial ênfase no contexto das substâncias lícitas, através de medidas de controlo sobre a publicidade e sobre a promoção de bebidas alcoólicas e tabaco, deve ser incentivada a fim de proteger toda a população com especial incidência, sobretudo grupos mais vulneráveis como os jovens.

O aumento do preço do álcool ou do tabaco através da aplicação de taxas, a regulação da disponibilidade física (como é o caso da limitação das licenças para venda de álcool em determinada área ou a proibição de venda a menores) ou a aplicação de penas (como é o caso da condução sob o efeito de álcool) são exemplos que demonstram ter uma relação directa com a redução dos problemas associados ao consumo. ${ }^{10,11}$

As evidências científicas sugerem que a adopção destas estratégias tem efeito no nível de consumo das substâncias e dos problemas com ela relacionados.

Nesta compilação de artigos acerca de Dependências apresentam-se dois estudos: um deles da autoria de André Reis ${ }^{12}$ e colaboradores e outro de Adriana Pinheiro e colaboradores, ${ }^{13}$ desenvolvidos em populações escolares, em que se caracterizam os consumos de álcool e de substâncias ilícitas e em que podemos encontrar prevalências destes fenómenos maiores que as prevalências de estudos nacionais neste âmbito. De acordo com o artigo de Luis Rebelo, ${ }^{14} \mathrm{o}$ médico de família, ao receber na sua consulta um paciente que fume, deve intervir usando a metodologia da Intervenção Breve (IB). Tendo por base a experiência do autor com pacientes fumadores, apresenta-se a aplicação prática da norma de orientação técnica para a cessação tabágica de Fiore e outros, tendo este artigo grande utilidade neste contexto clínico da Medicina Geral e Familiar portuguesa. 


\section{REFERENCIAS BIBLIOGRÁFICAS}

1. Rehm J, Room R, Monteiro M, Gmel G, Graham K, Rehn T, et al. Alcohol. In: World Health Organization, editor. Comparative quantification of health risks: global and regional burden of disease due to selected major risk factors. Geneva:WHO; 2004.

2. World Health Organization. Neuroscience of psychoactive substance use and dependence. Geneva:WHO; 2004.

3. Room R, Babor T, Rehm J. Alcohol and public health. Lancet 2005 Feb 5-11; 365 (9458): 519-30.

4. Hibell B, Guttormsson U, Ahlström S, Balakireva O, Bjarnason T, Kokkevi A. The ESPAD report 2003: alcohol and other drug use among students in 35 European countries. Stockholm: The Swedish Council for Information on Alcohol and Other Drugs; 2004.

5. Fiore MC, Bailey WC, Cohen SJ, et al. Treating tobacco and dependence: clinical practice guideline. Rockville, MD: US Department of Health and Human Services. Public Health Service; 2000.

6. Conigliaro C, Reyes C, Schultz J. Principles of screening and early intervention. In: Graham AW, Schultz TK, Mayo-Smith MF, Ries RK, Wilford BB, editors. Principles of addiction medicine. 3rd ed. Chevy Chase, MD: American Society of Addiction Medicine; 2003. p. 325-35.

7. Kaner EF, Beyer F, Dickinson HO, Pienaar E, Campbell F, Schlesinger C, et al. Effectiveness of brief alcohol interventions in primary care populations. Cochrane Database Syst Rev 2007 Apr 18; (2): CD004148.

8. Rebelo L. O médico de família e a dependência tabágica: uma oportunidade de ouro para intervir na qualidade de vida do doente. Rev Port Clin Geral 2004 Jan-Fev; 20 (1): 75-84.
9. Ribeiro C. Álcool: Impacto no indivíduo e na sociedade: qual o papel dos cuidados de saúde primários? Rev Port Clin Geral 2008 Mar-Abr; 24 (2): 269-74.

10. Babor TF, Caetano R, Casswell S, Edwards G, Giesbrecht N, Graham K, et al. Alcohol: no ordinary commodity - Research and public policy. Oxford: Oxford University Press; 2003.

11. Cunha Filho H. Necessidades de intervenção no consumo de álcool, tabaco e outras drogas em Portugal, Rev Port Saude Pública 2005 JunDez; 23 (2): 77-88.

12. Reis A, Barros J, Fonseca C, Parreira L, Gomes M, Figueiredo I, et al. Prevalência da INGestão de Álcool nos Adolescentes - Estudo PINGA. Rev Port Clin Geral. 2011 Jul-Ago; 27 (4): 338-45.

13. Pinheiro A, Picanço $P$, Barbeito J. A realidade do consumo de drogas nas populações escolares. Rev Port Clin Geral 2011 Jul-Ago; 27 (4): 348$-55$.

14. Rebelo L. Intervenção breve no paciente fumador: aplicação prática dos "5 As" e dos "5 Rs". Rev Port Clin Geral. 2011 Jul-Ago; 27 (4): 356$-61$

\section{ENDEREÇO PARA CORRESPONDÊNCIA}

\section{Cristina Ribeiro}

Faculdade de Medicina de Lisboa Instituto de Medicina Preventiva

Unidade de Medicina Geral e Familiar

cristina.mpr@sapo.pt 